\title{
STATUS OF THE HIPPARCOS DATA REDUCTION
}

\author{
J. Kovalevsky, J.L. Falin, M. Froeschlế and F. Mignard \\ OCA/CERGA \\ Avenue Copernic \\ 06130 Grasse, France
}

\section{PRESENT SITUATION OF THE SATELLITE}

HIPPARCOS is now well settled on its $10^{\mathrm{h}} 40^{\mathrm{m}}$ eccentric orbit and the data is received by three telemetry stations that are also transmitting commands: Odenwald (F.RG.), Perth (Australia) and Goldstone (Californla). Although during about 80 to $90 \%$ of the time, data are recovered, in practice only the observations made from higher than the outer Van Allen belt are usable. Sometimes even at that height, on-board attitude determination does not converge due to the noise that covers the star mapper signal. Finally, about between 55 and $65 \%$ of the mission time is usable for reduction. The actual routine data recovery started on November 27, 1989.

\section{WORK OF THE REDUCTION TEAMS}

Both data reduction consortia (FAST and NDAC) have received and reduced about 1,5 months of data sampled thoughout the first eight months of mission. The main objectives were :

- To modify the software in order to accomodate the new situation arising from the unexpected orbit and the increased noise when the satellite is still close to the radiation belts or when it is in lengthy occultation situation. More generally, the treatment of the real data often showed features that were not correctly dealt with by software built using some a priori model.

- To calibrate the instrument: intensity and modulation transfer functions, grid to field and field to grid transformation, basic angle, etc...

- To test the correctness of the software by comparison between various intermediary results obtained by the two consortia.

This initial phase is essentially complete, and it expected that routine data reduction might start sometime in November 1990. It should last at least 4 years, possibly more if the duration of the mission is extended.

\section{PRECISION OF THE RAW DATA}

Raw data consists of photo-electron counts produced by the photomultipliers. The reduction using a calibrated model gives the times of crossings of the star mapper grids 
and the modulation phases that are used to determine the position of a star image on the main grid. Both can be readily transformed into milliseconds of arc on the sky (fig. 1 and 2).

In addition, the amplitude of the modulation curve on the main grid gives the intensity of the light recelved from the star and, hence, its magnitude in the HIPPARCOS photometric system. Using as calibrators a certain number of standard stars that have been accurately observed from the ground. one can dertve the accuracy of magnltude determinations in addition to their precision (see fig. 3). Actually, there are two independent methods to determine the magnitude using different terms in the modulation function. They give the same results for single stars, but there are differences in case of non-single stars and this is one of the ways to identify double or multiple stars.

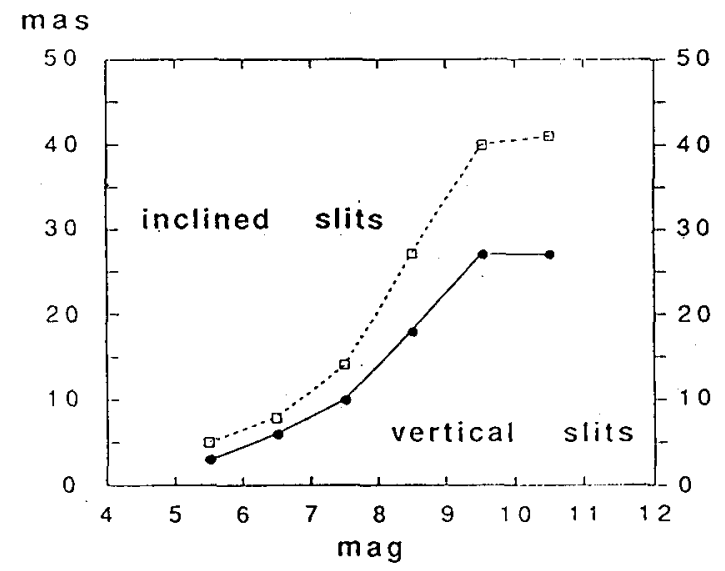

Eigure 1 - Typical average r.m.s. errors of the determination of the times of transit of a star through vertical and inclined slits of the star mapper in function of magnitudes.

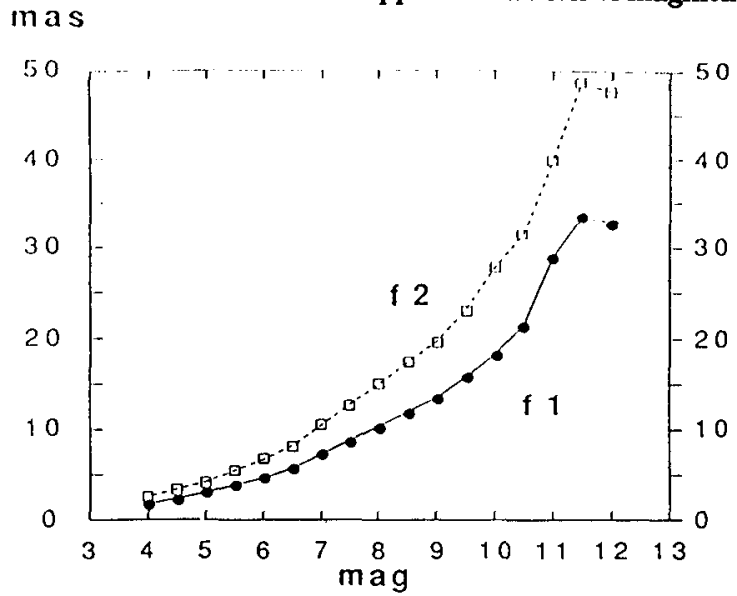

Elgure 2 - Typical average r.m.s. errors on the determination of the phases of the first and second harmonic of the modulated signal from the main grid in functlon of magnitudes. The corresponding observation times range from $0.2 \mathrm{~s}$ for bright stars to 1 second or more for the fainter ones. 


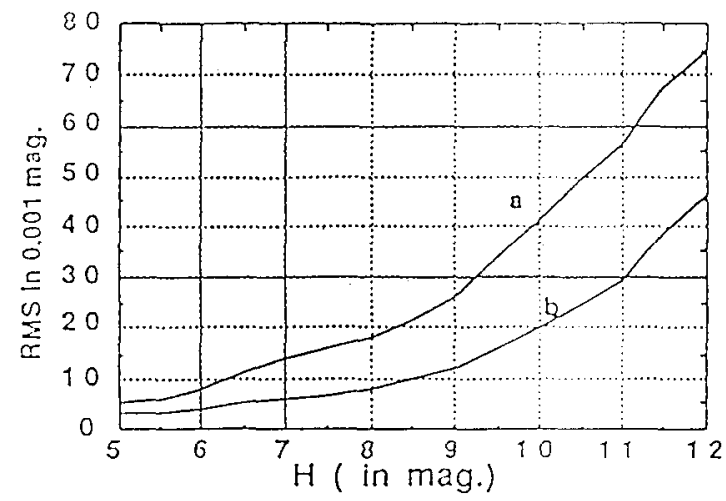

Figure 3 - Standard deviation per transit as a function of the magnitude for the two methods (a), (b) of determining it.

Up to a couple of dozen new double stars are discovered by this methods from the analysis of 5 to 8 hours of data. The separation power depends of course on the magnitude difference between the components. In the most favourable cases it could be as low as $0 " .05$.

\section{REDUCTION ON A GREAT CIRCLE}

The reduction procedure calls for a synthetic treatment of the results of the raw data analysis presented in the preceding section, as accumulated during a data set consisting of the useful part of the satellite revolution. Two steps are performed.

\section{a) Attitude determination from the star mapper}

About 3000 to 6000 transit times through each group of slits are obtained. The attitude is determined (this is a first approximation) using the star positions provided by the Input Catalogue. The following r.m.s. are typically obtained for the three orientation angles :

$$
\begin{aligned}
& \psi: 60 \text { to } 100 \text { mas (along track) } \\
& \theta: 100 \text { to } 150 \text { mas (around oy axis) } \\
& \phi: 200 \text { to } 400 \text { mas (around ox axis) }
\end{aligned}
$$

However, these precisions are primarily affected by the uncertainties of the Input Catalogue. The attitude so obtained may be used to improve the along scan positions deduced from the Input Catalogue. The results show that indeed the catalogue induced errors are of the order of the attitude error obtained. It also shows the quality of the Input Catalogue which is of the order of $0^{\prime \prime} .2$ to $0 " .3$.

\section{b) Abscissae on the reference great circle}

This last global step in the data set reduction is the determination of the mean abscissa of each observed star on the reference great circle using the attitude and the grid coordinates provided earlier. Simultaneously, the $\psi$ component of the attitude is redetermined as well as some instrumental parameters. A typical r.m.s. of the order of 10 to 16 mas characterizes the precision of the abscissae on the great circle. The same precision is obtained for the along scan attitude $\psi$. 


\section{EINAL PRECISION EXPECTATIONS}

The determination of positions, proper-motions and parallaxes requires a new step in which the results obtained for a large number of reference great circles are combined. This could not be done since too few data have been reduced so far. However, it is possible to extrapolate the precisions presented above using our experience with simulated data. In particular we can use the fact that these positions are better than the nominal ones, and this partially compensates the loss of data acquisition. Table 1 gives the expected precisions as a function of the duration of the mission.

The behaviour of the satellite proves to be such that a 4-year duration is not to be excluded. In addition, one estimates that various improvements of the reduction method would improve the results by a factor of 0.7 . From this we conclude that reaching the nominal precisions (and seemingly also accuracies) is a reasonable expectation if the satellite survives another couple of years. If, as it is quite possible, finances permitting. HIPPARCOS remains absolutive another couple of years, a final precision of the order of one millisecond of arc is not impossible.

\section{TABLE I}

Expected precisions in m.a.s. for a 9 magnitude star

\begin{tabular}{|l|c|c|c|c|c|c|}
\hline \multicolumn{1}{|c|}{$\begin{array}{c}\text { Duration of } \\
\text { the mission }\end{array}$} & 18 & 24 & 30 & 40 & 50 & $\begin{array}{c}\text { Nominal } \\
\text { mission } \\
\text { (30 months) }\end{array}$ \\
\hline Position & 6 & 4.5 & 3 & 2 & 1.5 & 2 \\
$\begin{array}{l}\text { Annual proper } \\
\text { motions }\end{array}$ & 10 & 4 & 2.5 & 1.7 & 1.0 & 2 \\
Parallaxes & 6 & 4.5 & 3 & 2 & 1.5 & 2 \\
\hline
\end{tabular}

\section{MAIN LITTERATURE}

The HIPPARCOS Mission, Prelaunch status.

Vol, 1: "The HIPPARCOS satellite", M.A.C. Perryman, H. Hassan and 22 other authors, 353 pages.

Vol 2: "The Input Catalogue", M.A.C. Perryman, C. Turon and 21 other authors, 290 pages.

Vol3: "The Data Reduction", M.A.C. Perryman, L. Lindegren, C.A. Murray, E. Hoeg, J. Kovalevsky and 49 other authors, 515 pages.

ESA Publication SP1111, July 1989. 